\title{
The Potential Use of Insulin-like Growth Factor I (IGF-I) as a Neuronal Rescue Therapy
}

\author{
Peter D. Gluckman, Jian Guan, Erica Beilharz, Barbara M. Johnston, Odette Miller and Chris Williams \\ Research Centre for Developmental Medicine and Biology, University of Auckland, Auckland, New Zealand
}

Asphyxial or ischemic insults to the brain lead to neuronal loss in two phases-one associated with the insult and a second phase which is delayed for some hours after the injury, termed delayed neuronal death (DND). Recent evidence suggests that provided the initial insult is not excessively prolonged, DND is a major component. The mechanisms of DND are incompletely resolved but include apoptosis. Because of the delay in the onset of DND (generally to 8-72 hours after an insult) there is a window of opportunity in which it may be possible to block the processes initiated by asphyxia that lead eventually to DND. Using a standardised model of hypoxic ischemic injury (HII) in the 21 day rat we have shown using in situ hybridisation that insulinlike growth factor I (IGF-I) IGFBP-2 and IGFBP-3 are induced in regions of neuronal loss within 1 to 3 days of injury; induction is seen both in areas of infarction and of selected neuronal loss. In contrast IGF-II is not induced until 7 days after injury and is restricted to the area of infarction. We therefore examined, in the adult rat with unilateral fill, the effect of IGF-I administration via the lateral cerebral ventricle. IGF-I given prior to the insult had no effect. However IGF-I (5 to 50 $\mu \mathrm{g})$ given as a single injection 2 hours after the injury markedly reduced $(\mathrm{P}<0.05)$ the incidence of infarction and the degree of neuronal loss throughout the injured hemisphere. The histological improvement was associated with improved functional outcome as measured by bilateral tactile responses. Co-administration of IGF-II blocked the effect of IGF-I suggesting that IGF-I was attracted to the region of injury by IGF binding proteins and that this is blocked by saturation with IGF-II. Similarly des-IGF-I was not effective at equimolar doses to IGF-I, perhaps reflecting reduced association with IGFBPs. Alternatively, or in addition, IGF-I may act in part by being the precursor for the $\mathrm{N}$ terminal tripeptide, GPE which has some neuroactivity. We examined the effect of GPE $(3 \mu \mathrm{g})$ administered similarly 2 hours after HII and found significant $(\mathrm{P}<0.05)$ neuroprotection extending throughout the injured hemisphere. The mode of action of GPE is not clear as the effect is not mimicked by MK801, a NMDA receptor antagonist. However it seems unlikely that the entire effect of IGF-I is to act as a precursor for GPE given the induction of IGF receptors after injury and the neuroprotective effect of high doses of insulin. We have further examined the neuronal rescue action of IGF-I in fetal sheep subject to reversible cerebral ischemic injury. A single dose of $100 \mathrm{ng}(\mathrm{n}=6)$ or $1 \mu \mathrm{g}(\mathrm{n}=7)$ given 2 hours after injury conferred marked neural protection $(\mathrm{P}<0.05)$. Thus in two species with markedly different models of brain injury IGF-I is neuroprotective when given after the injury. We speculate that IGF-I acts to interfere with processes leading to the induction of apoptosis. These studies suggest the possibility of the therapeutic use of IGF-I as a neuronal rescue therapy. 